\section{A Multi-National Human- Computer Interaction Evaluation of the Public Participatory GIS GeoCitizen}

Gl_Forum 2019, Issue 1

Page: 18 - 39

Full Paper

Corresponding Author: mona.bartling@sbg.ac.at

DOI: 10.1553/giscience2019_01_s18

\author{
Mona Bartling1, Bernd Resch1', Anton Eitzinger² and Leo Zurita-Arthos ${ }^{3}$ \\ 'University of Salzburg, Austria \\ International Center for Tropical Agriculture (CIAT), Colombia \\ 3Universidad San Francisco de Quito (USFQ), Ecuador
}

\begin{abstract}
Designing user-friendly Public Participatory Geographic Information Systems (PPGIS) is a challenging task, since a PPGIS is typically used by users who have different characteristics and different requirements and needs. Hence, applying Human-Computer Interaction $(\mathrm{HCl})$ principles is of particular importance in designing PPGIS. This study aims to create an inventory of usability aspects of a PPGIS by focusing on understanding the characteristics of a broad range of users. The usability study included 73 participants from Colombia, Uganda and Austria. We combined a custom qualitative survey (conducted in all three countries) with an eye-tracking based survey (conducted only in Austria). Considerable usability problems were faced especially by participants with low levels of IT-literacy. This was mostly due to a lack of experience in using functionally complex smartphone applications or interactive maps. In general, we observed a high level of difference in usability between the user groups. The eye-tracking statistics for the Austrian study supported the outcomes of the qualitative survey well.
\end{abstract}

\title{
Keywords:
}

mobile PPGIS, usability, multi-national study, eye-tracking

\section{Research in Human-Computer Interaction and Public Participatory Geographic Information Systems}

Public Participatory Geographic Information Systems (PPGIS) are commonly used in spatial planning processes where different kinds of users are involved. PPGIS are designed to support decision-making processes that involve citizens, taking their opinions into account (Corbett \& Keller, 2005; Irvin \& Stansbury, 2004; Stivers, 2016). PPGIS are typically used by a heterogeneous user group, e.g. having different levels of experience, skills and interests (Brown et al., 2013). Some potential user groups are not used to working with this kind of application. Consequently, a PPGIS should be designed in such a way that all users can easily and enjoyably interact with it (Sidlar \& Rinner, 2007; Tobón \& Haklay, 2002). Thus, it is particularly important for the design of a PPGIS to include Human-Computer Interaction 
(HCI) principles, and to investigate the usability of the PPGIS (Brown et al., 2013; Tobón \& Haklay, 2002).

A number of HCI design principles are addressed in the PPGIS literature. Meng and Malczewski (2009) state that the map interface should highlight specific elements, and that the number of buttons and amount of information should be limited. Timita (2014) describes how any piece of information or visual element should be carefully chosen and should not overload the user. Skarlatidou and Haklay (2006), and López-Ornelas, AbascalMena and Zepeda-Hernández (2013) indicate that the map presentation, colours and other features affect the user's perception and should be evaluated according to the application's objectives. Newman et al. (2010) also discuss the importance of explaining the context and purpose of the application to the user thoroughly. López-Ornelas et al. (2013) further state that each symbol should be intuitive and easily understandable, so that the user can concentrate on the essential information without being distracted by having to read explanations for the symbols. They also show that inexperienced users prefer to use map applications that are in a pre-defined state and offer all the information needed at once. A study by Brown et al. (2013) re-emphasizes Nielsen and Mack's guideline (1994) that natural terminology should be used. Lastly, Newman et al. (2010) mention that a 'motivating' application design creates a positive and pleasant experience.

Usability studies help to evaluate these design principles and their influence on users. Numerous usability studies have been conducted for PPGIS (e.g. Butt and Li (2015), Haklay and Tobón (2003), Meng and Malczewski (2009), Poplin (2015), Skarlatidou and Haklay (2006), and Atzmanstorfer et al. (2016)). These studies are usually carried out with participants who have similar user characteristics. We argue that PPGIS are often used in quite different geographical contexts with different user types. Hence, we see a gap in the literature analysing the parameters and consequences of user characteristics (in different geographical regions), and how these affect the usability of a PPGIS.

This paper therefore aims to evaluate the usability aspects of a mobile PPGIS for participants with a wide range of characteristics, including their varying IT skill levels and age. We asked people from three different areas - Colombia, Uganda and Austria - to participate in a qualitative survey to evaluate the usability of a PPGIS. Further, like Çöltekin, Heil, Garlandini and Fabrikant (2009), we combined a custom qualitative survey with eyetracking statistics as an objective way of measuring usability aspects. Since the eye-tracking device was not available in Colombia and Uganda, we were able to combine the qualitative survey with eye-tracking only for the participants from Austria.

The paper addresses the following research questions:

- What are the key usability problems of a PPGIS for participants in a multi-national study that includes a broad diversity of user types?

- To what degree do these main usability problems depend on age, IT-literacy, national context and type of task?

- Do eye-tracking statistics support the findings of the qualitative survey? 


\section{Study Design}

This usability study evaluates the GeoCitizen application as an example of a PPGIS (Figure 1). The application aims to structure citizens' knowledge about local phenomena for use in the spatial-planning decision-making process in cities and municipalities. Citizens can participate in surveys, upload georeferenced ideas for improving their environment, and involve themselves in discussions with, for example, their neighbours (Atzmanstorfer, Resl, Eitzinger, \& Izurieta, 2014). The application was developed by the Fundación CEC in cooperation with the Interfaculty Department of Geoinformatics - Z_GIS, University of Salzburg, and the International Center for Tropical Agriculture (CIAT), Colombia.

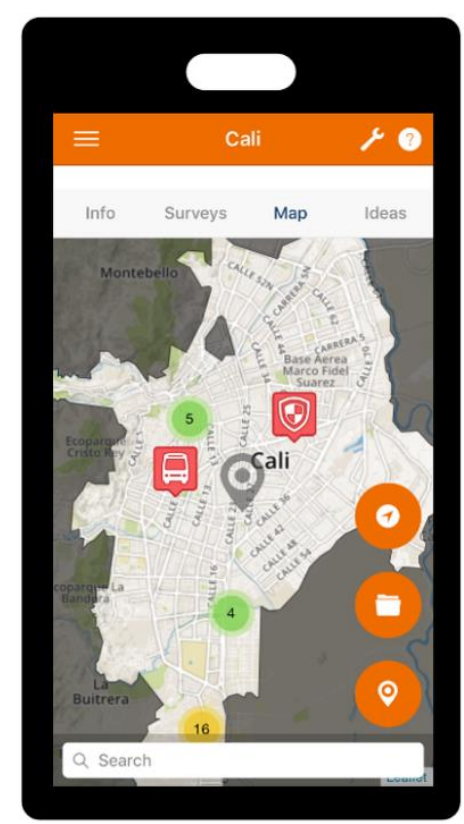

Figure 1: GeoCitizen Application

Atzmanstorfer et al. (2016) conducted a usability study using GeoCitizen with participants from marginalized communities in Cali, Colombia. This paper extends their approach by testing the usability with novice users of different user characteristics from three study areas. We measured the effectiveness, efficiency and satisfaction of using the PPGIS. This corresponds to ISO standard 9241-11 defining usability (International Organization for Standardization, 1992). The three aspects of usability refer to the ease, pleasure, comfort and trust experienced when carrying out tasks with an application (Nayebi, Desharnais \& Abran, 2012).

In this study, participants carried out six tasks (Table 1). Two of these concerned general interaction with the application (entering the application; changing the profile configuration of the account). The remaining tasks were about interacting with the map element (changing the basemap; finding and reviewing a Point of Interest (POI); adding a new POI). We 
measured three usability metrics of the application - effectiveness, efficiency and satisfaction. Effectiveness corresponds to the error rate: completion without errors, with light non-critical errors (slight usability problems that were overcome), moderate non-critical errors (major usability problems that were overcome), or critical errors (major usability problems that were not overcome). Efficiency corresponds to the time spent on each task. Finally, we measured the participants' satisfaction. After participants had completed the tasks, we asked general questions about the application's design so as to understand usability in a more holistic manner. Remarks and observations were documented while the participants were carrying out the tasks and/or responding to the questions.

Table 1: Survey Design

\begin{tabular}{|c|c|}
\hline \multirow{6}{*}{ Task scenario } & (1) Enter the application and navigate to the map \\
\hline & (2) Change the basemap \\
\hline & (3) Find a POI and review its description \\
\hline & (4) Leave a comment on a POI \\
\hline & (5) Add a new POI \\
\hline & (6) Find the profile configuration and change the age \\
\hline \multirow{2}{*}{$\begin{array}{l}\text { Application design } \\
\text { evaluation }\end{array}$} & General remarks \\
\hline & Design evaluation \\
\hline
\end{tabular}

In addition, we used an eye-tracking device for the Austrian participants. As Poole and Ball (2005) indicate, statistics derived from eye-tracking aim to understand the cognitive processes of the participants. This in turn supports the evaluation of usability aspects (Çöltekin et al., 2009).

In the eye-tracking based evaluation, the application's interface was defined by and divided into Areas of Interests (AOI) for which it was possible to obtain statistics, such as when, for how long and how often eye fixations occur on an AOI. Our study included two eyetracking metrics: (1) the time to first glance at the target AOI (i.e. the AOI that the participant had to focus on in completing the task), compared to how long it took to complete the task, indicating how much attention the AOI drew, and how clear the task to be completed was to the participants; (2) comparing the number of fixations on the target AOI to the total number of fixations reveals how search-efficient the participants were in completing the tasks. A higher number of fixations equals lower search-efficiency (Çöltekin et al., 2009; Poole \& Ball, 2005).

We selected 73 participants from three different study areas: 30 from Colombia (12 female, 18 male), 23 from Uganda (9 female, 14 male), and 20 from Austria (11 female, 9 male). (See Appendix 1 for a detailed overview of participants' attributes.) In selecting countries of differing levels of economic development, we expected to find participants with varying user characteristics. The participants from Colombia were students at Camacho University in Cali, and employees from CIAT in Palmira. In Uganda, the participants were coffee farmers from 
the Luweero district, students from the Makerere University in Kampala, and employees from the International Institute of Tropical Agriculture (IITA) in Kampala. In Austria, students and employees from the University of Salzburg participated. The participants were invited by email and assigned a time slot. They were divided into four different user groups according to age range and self-rated IT skills (Table 2). For coherence, the same age ranges were selected as in the earlier usability study by Atzmanstorfer et al. (2016).

Table 2: User Groups and Number of Participants

\begin{tabular}{|l|l|l|l|}
\hline Age/IT-literacy & Colombia & Uganda & Austria \\
\hline 18-29 years/low-medium & 8 & 5 & 5 \\
\hline 30-65 years/low-medium & 7 & 8 & 5 \\
\hline 18-29 years/medium-high & 8 & 5 & 5 \\
\hline 30-65 years/medium-high & 7 & 5 & 5 \\
\hline
\end{tabular}

We asked the participants to sit at a desk in a quiet place. In Austria, the participants were linked to the eye-tracking device, which was installed on the desk. In all study areas, the smartphone remained on the desk, without being moved or held by the participants. Each survey took between 15 and 20 minutes, depending on the amount of feedback that the participants provided. For each task, we gave oral instructions. If clarification was needed, we allowed participants to ask questions. Since most participants were unfamiliar with GISrelated terms (e.g. basemap, POI), we explained these using language that was accessible to the participant.

\section{Results}

\subsection{Qualitative Survey}

Figure 2 and Figure 8 show the error rate (effectiveness) of tasks 1 and 6, concerning general interaction with the application. Figure 3, Figure 5, Figure 6 and Figure 7 show tasks 2-5, which relate to interactions with the map.

For task 1 (Figure 2), the participants were asked to navigate to the map. The majority of the participants were able to complete the task without help. Participants from Austria and Colombia finished without errors or with non-critical errors (mostly light non-critical); medium-high IT-literate participants from Uganda finished the tasks without errors; Ugandan participants of low-medium IT skills experienced greater difficulty, for the most part completing the task with non-critical errors, while $20 \%$ of Ugandan participants aged 18-29 finished with critical errors. Nevertheless, for the majority of participants the interface design was intuitive. 


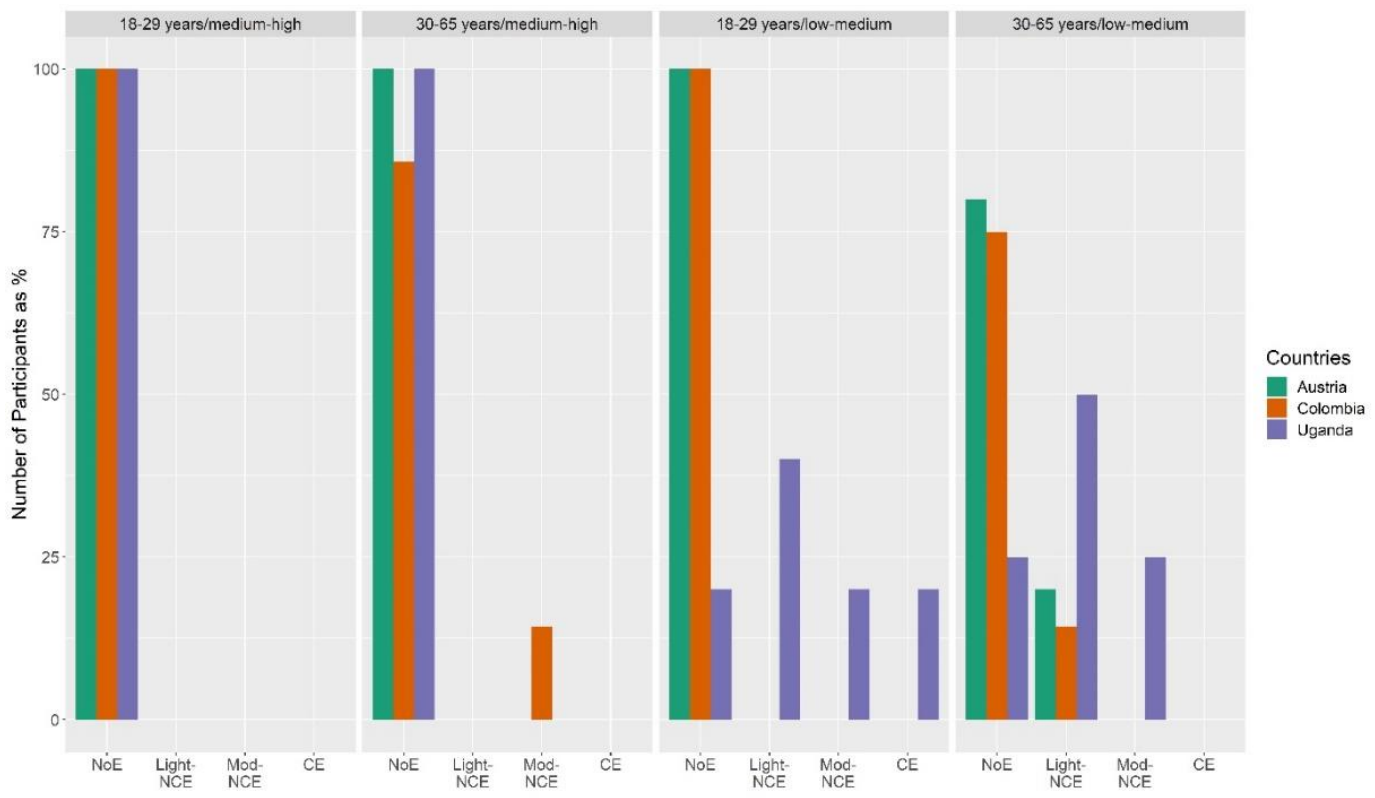

Figure 2: Error Rate (Effectiveness) of Participants for Task 1 (Navigating to the Map): NoE $=$ No Errors, Light-NCE $=$ Light Non-Critical Errors, Mod-NCE $=$ Moderate Non-Critical Errors, CE $=$ Critical Errors

Task 2 (Figure 3) asked the participants to change the basemap. Several participants were not used to the concept of modifying a basemap. Low-medium IT-literate participants in particular faced problems while carrying out the task, indicated by the higher number of critical errors. None of the Ugandan and Colombian participants of the user group 30-65 years with low-medium IT skills was able to carry out the task. Most Austrian and Colombian participants with medium-high IT skills performed the task without errors or with non-critical errors. 
Bartling et al

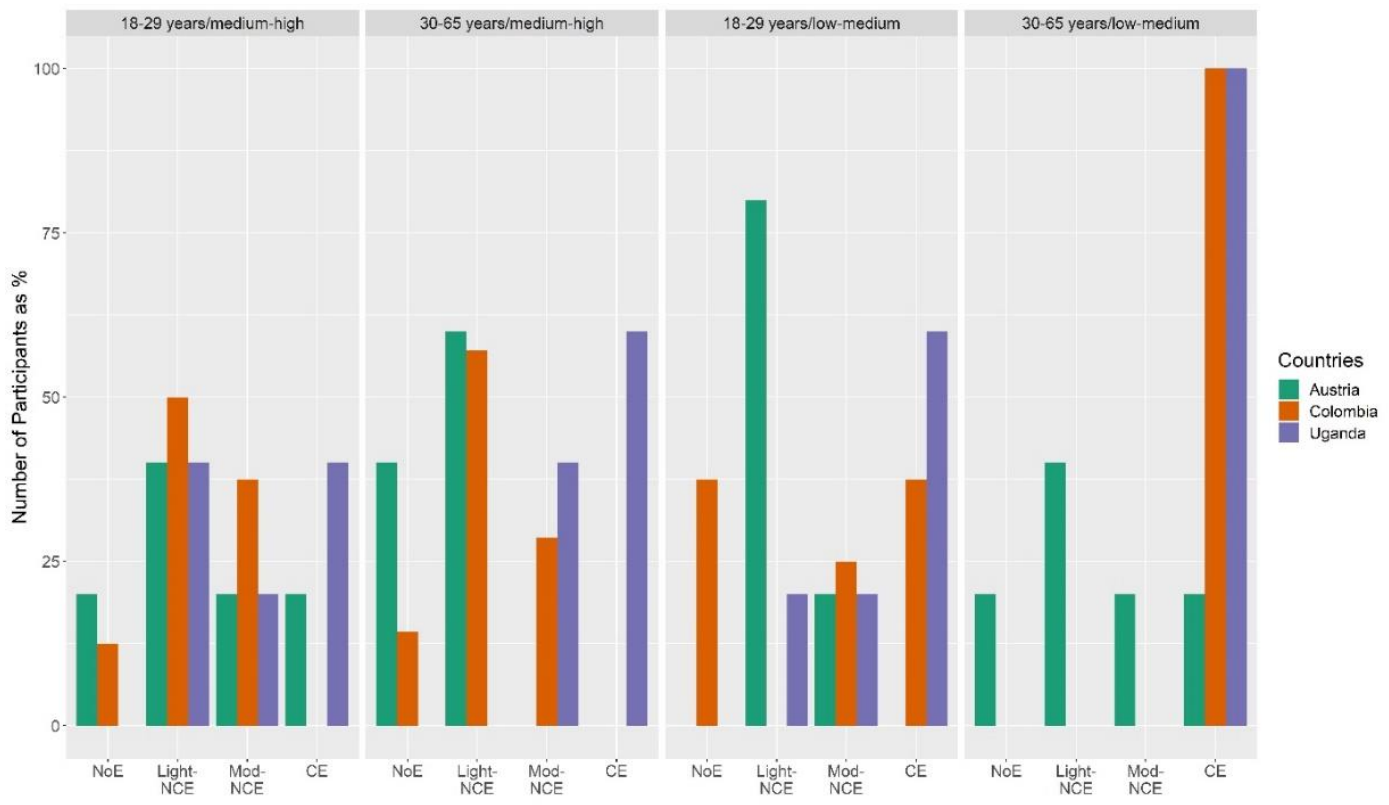

Figure 3: Error Rate (Effectiveness) of Participants for Task 2 (Change the Basemap): NoE $=$ No Errors, Light-NCE $=$ Light Non-Critical Errors, Mod-NCE $=$ Moderate Non-Critical Errors, $C E=$ Critical Errors

Many participants had difficulties understanding the meaning of the button icons. Inside the map are three buttons that can be tapped to open a menu of additional buttons (Figure 4).



Figure 4: Button Groups 
Most of the participants were guessing which button was the right one for the task, randomly screening, tapping and/or selecting other buttons on the map, thus indicating that the icons do not clearly illustrate the buttons' functionalities.

For task 3 (Figure 5), participants had to find a POI on the map and review its description. Most of the participants did not use the search bar which had been designed for this purpose. We deduce from this that the search bar must be better placed. Some of the participants discovered and used it after a while; others found the right POI by chance; some were not able to complete the task at all. Generally, we detected a high number of critical errors among participants from all study areas, except for participants aged group 18-29 years with medium-high IT skills.

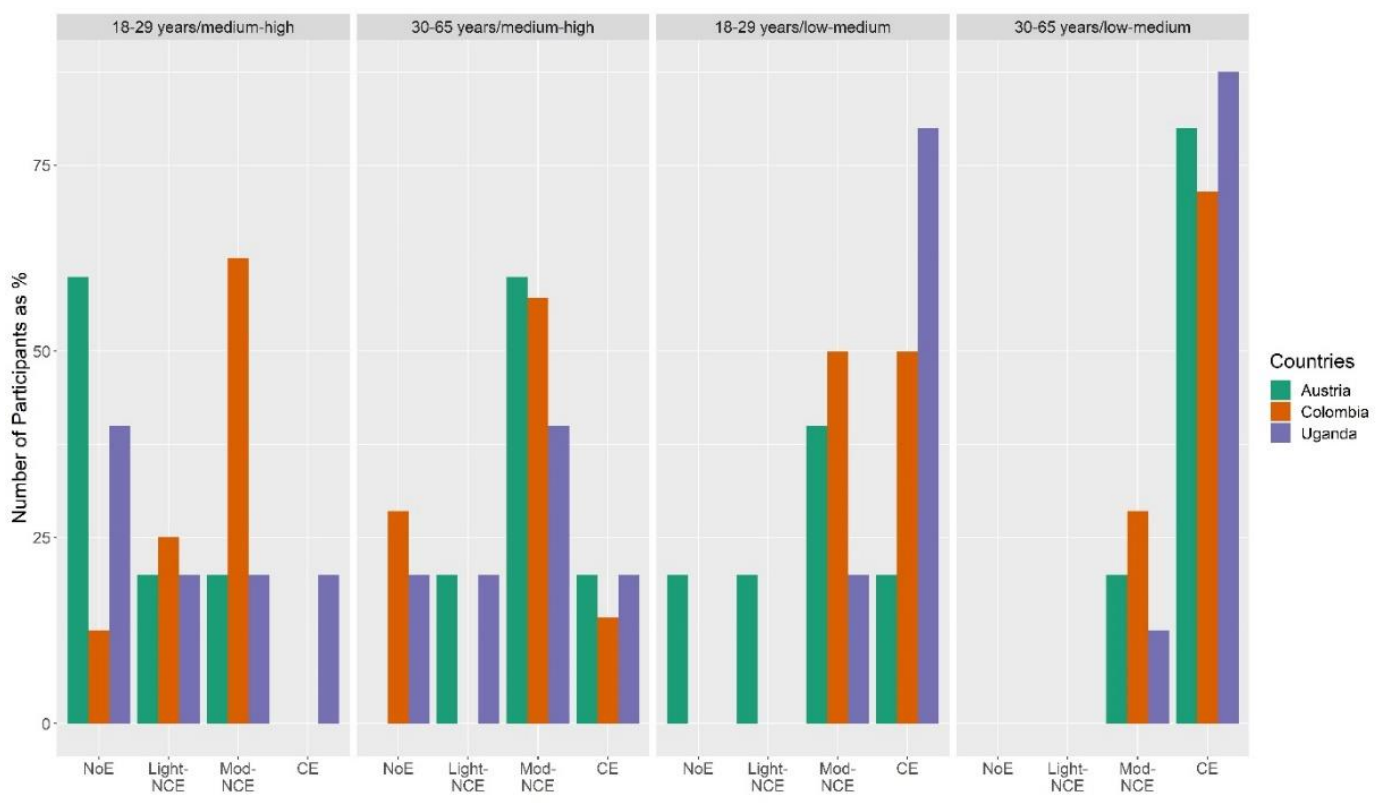

Figure 5: Error Rate (Effectiveness) of Participants for Task 3 (Find and Review Description of a POI): NoE $=$ No Errors, Light-NCE $=$ Light Non-Critical Errors, Mod-NCE $=$ Moderate Non-Critical Errors, CE $=$ Critical Errors

For task 4 (Figure 6), participants had to leave a comment on an existing POI. For most, this task was easy to complete. The majority of the medium-high IT-literate participants from Colombia and Austria completed the task without any errors or with light non-critical errors. Most of the Ugandan participants of the same user profiles had a higher number of moderate non-critical or critical errors. Most of the Ugandan low IT-literate participants of the 18-29 age group completed the task with moderate non-critical errors, the rest with critical errors. Most of the Colombian participants of the same user group finished without errors; however, $25 \%$ of the participants made critical errors. Austrian participants with low IT-literacy showed mixed results. While the younger user group completed the task mostly without errors, the older user group, with $40 \%$, had the highest number of critical errors of 
all Austrian participants. Most of the Colombian and Ugandan participants aged 30-65 with low-medium IT skills completed the task with critical errors.

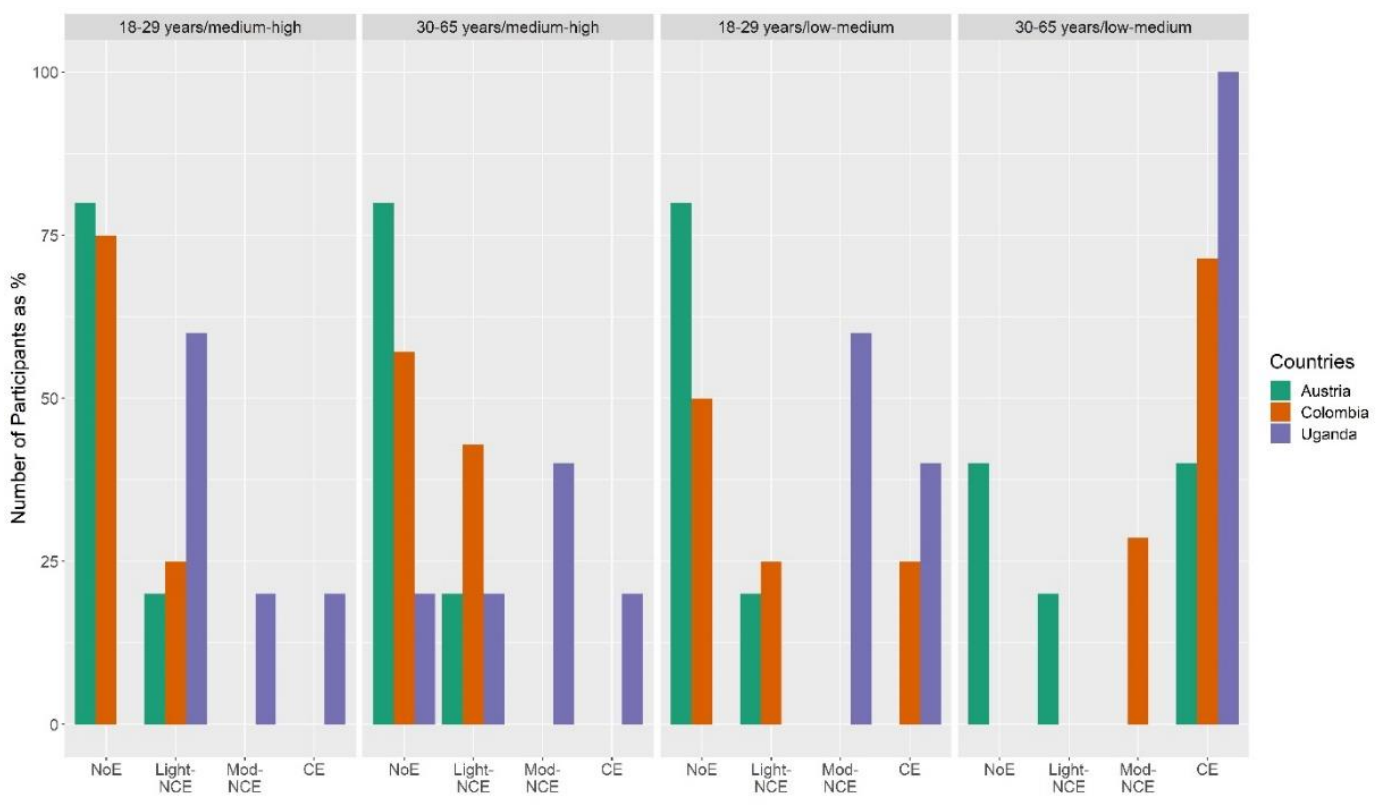

Figure 6: Error Rate (Effectiveness) of Participants for Task 4 (Add Comment on an Existing POI): NoE = No Errors, Light-NCE $=$ Light Non-Critical Errors, Mod-NCE $=$ Moderate Non-Critical Errors, $C E=$ Critical Errors

From our observations, the location of the information seemed to be intuitive to most of the participants, and the meaning of the button icons was understood. The participants who made critical or moderate non-critical errors had already faced usability problems in the previous tasks. We assume that these participants had a higher level of frustration that decreased their motivation to complete the task. This is a crucial insight that underlines the importance of a frustration-free experience.

For task 5 (Figure 7), we asked the participants to add a new POI. Austrian and Colombian participants of the user group 18-29 years with medium-high IT skills completed the task without errors or with light non-critical errors. Austrian participants of the other user groups mostly carried out the task without errors or with light non-critical errors. Only participants aged 30-65 with low-medium IT skills made critical errors $(20 \%)$. Colombian participants aged 30-65 with medium-high IT skills for the most part carried out the task with moderate non-critical errors (43\%); $29 \%$ of this user group were unable to finish the task. The majority of low IT-literate participants from Colombia were unable to complete the task $(50 \%$ and $71 \%$ for the younger and older age groups respectively). A high number of Ugandan participants from almost all user groups were unable to finish the task, with $60 \%, 80 \%$ and $50 \%$ of critical errors for the user groups aged 30-65 with medium-high IT skills, and both age groups of low-medium IT skills. Only $60 \%$ of the participants of the user group 18-29 years with medium-high IT skills were able to finish the task with light non-critical errors. 


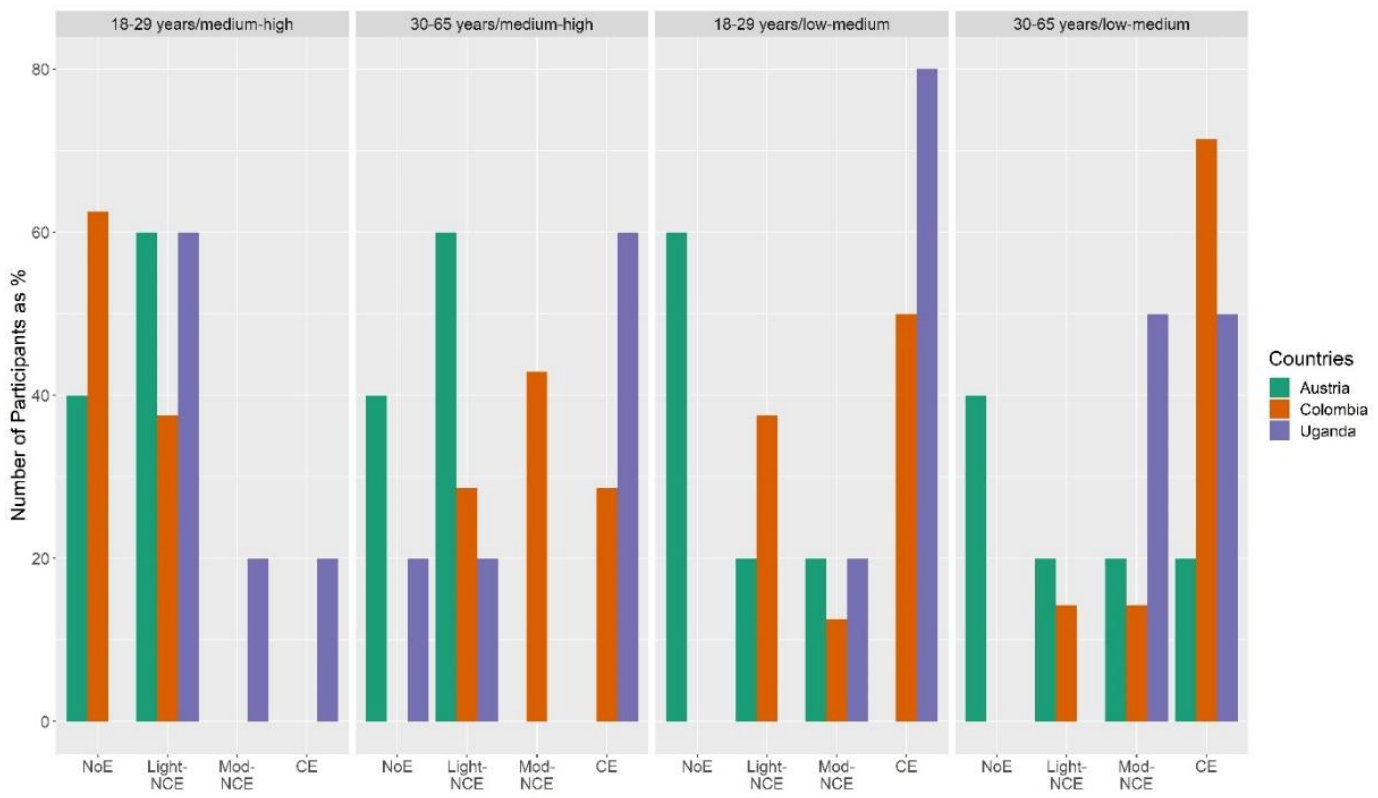

Figure 7: Error Rate (Effectiveness) of Participants for Task 5 (Adding a POI): $\mathrm{NoE}=$ No Errors, Light-NCE $=$ Light Non-Critical Errors, Mod-NCE $=$ Moderate Non-Critical Errors, CE $=$ Critical Errors

As in task 2, some participants faced problems finding the right button. In order to add a new POI, there is a marker positioned in the centre of the map that the participants have to place over a selected location. Afterwards, a tap on a button opens a menu to input the POI description. Some participants were confused by the marker in the centre of the map; some expected to have to tap for longer on a specific location in order to create a new POI; others thought that they should first tap on the button for adding a POI and then define the location. Hence, we need to understand what seems to be the most intuitive way to add a new POI and to re-design this functionality.

For the last task (Figure 8), we asked the participants to make changes to the profile configurations of the account. Here, they faced considerable problems completing the task. Most of them criticized the fact that the profile configuration was hidden. Apart from 12.5\% and $20 \%$ of the Colombian and Ugandan participants belonging to the user group aged 1829 with medium-high IT skills, participants were unable to carry out the task without any errors. The user group of those aged 30-65 with low-medium IT skills faced the highest number of problems. Here, all participants from Colombia and Uganda and $80 \%$ of the participants from Austria made critical errors. The rest of the Austrian participants finished the task with moderate non-critical errors. The Ugandan participants had the most difficulties, followed by the Colombian participants. Comparing the user groups, we detected a trend (reading the diagram from left to right): fewer usability problems were encountered by the user group aged 18-29 with medium-high IT skills, and more were found by the user group aged 30-65 with low-medium IT skills. 


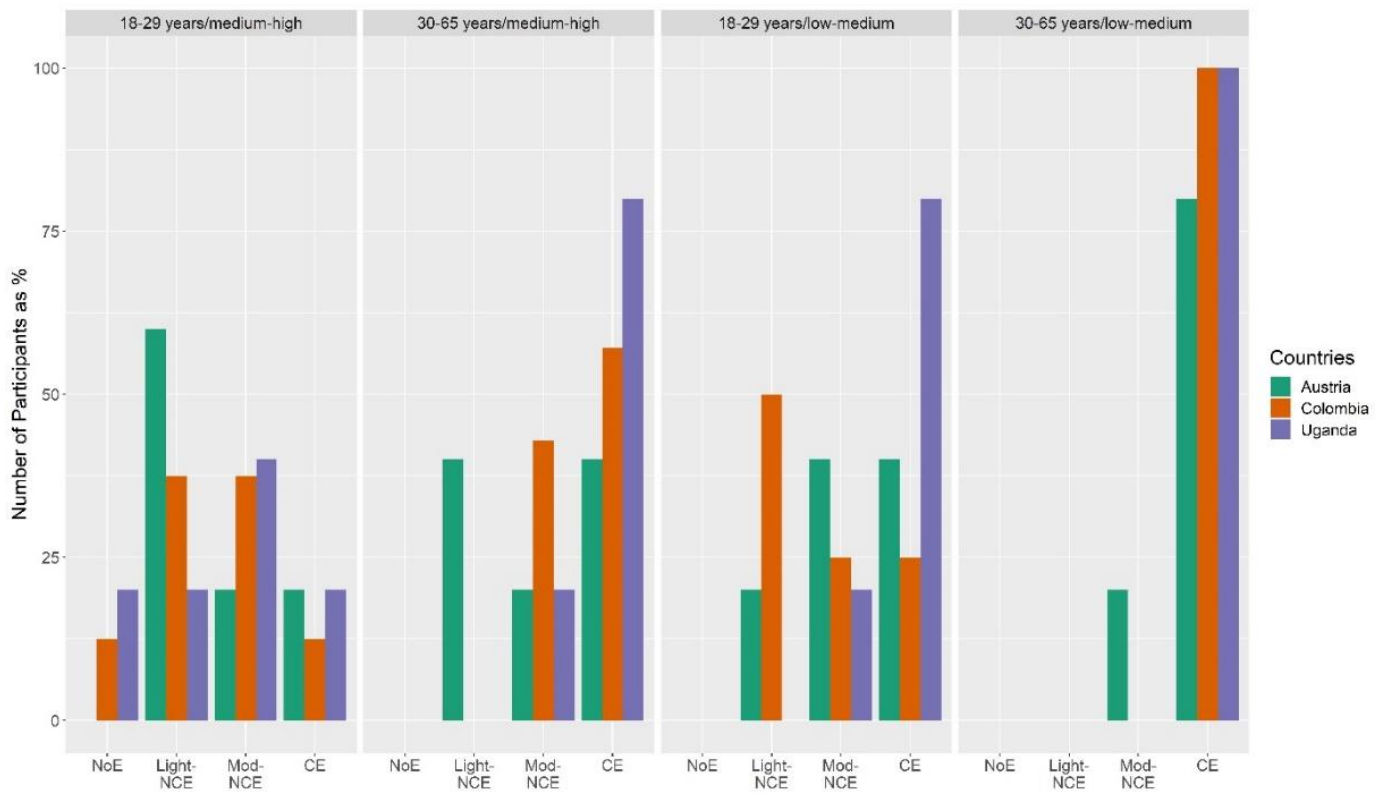

Figure 8: Error Rate (Effectiveness) of Participants for Task 6 (Reconfiguring the Profile): NoE = No Errors, Light-NCE $=$ Light Non-Critical Errors, Mod-NCE $=$ Moderate Non-Critical Errors, $C E=$ Critical Errors

We hypothesize that the high number of moderate non-critical errors and critical errors was due to two problems. First, the application offers three separate configuration menus for the information/visualization settings, basic application settings (e.g. language settings) and the user-profile information (Figure 9).
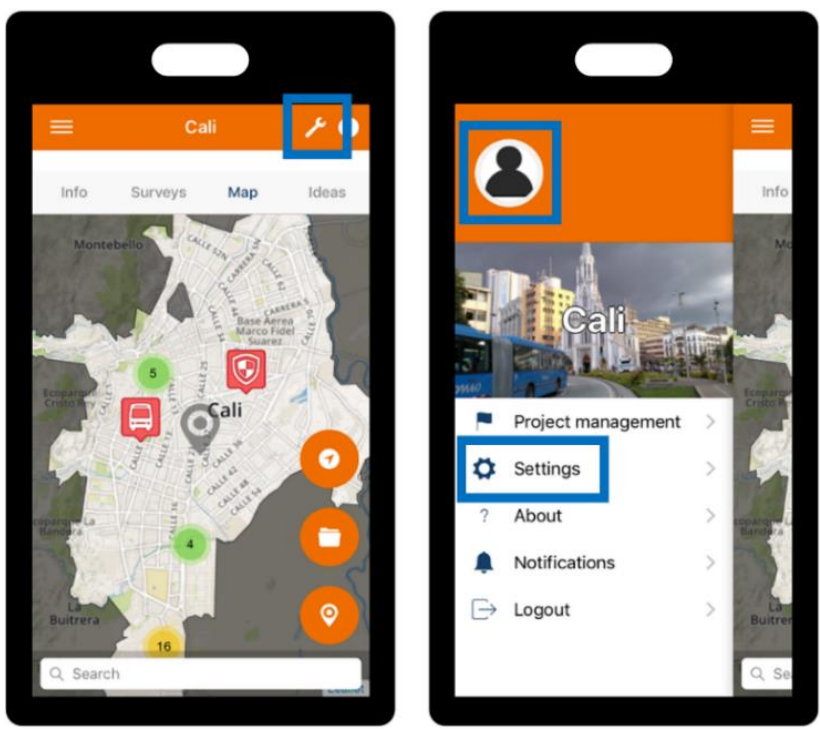

Figure 9: Configuration Elements of Taskbar (Left) and Side Menu (Right) 
The first two configurations were prominently displayed in the side menu or in the taskbar menu. Hence, participants were distracted by these menus and did not find the profile configurations. Second, the profile configurations are accessible by tapping on the user profile icon in the upper section of the side menu. However, the account did not reflect the participant's name, since we provided a neutral account for the study. We deduce that due to the other two configuration options and the neutral profile, participants got confused. In order to avoid this, we could have asked participants to create their own accounts to be used for the task scenario. However, the application needs a reliable internet connection for the creation of an account, which was not the case in Uganda and, therefore, we chose to provide an account for all participants.

For the qualitative study, we also measured the time spent on each task (efficiency) and the evaluation by the participants (satisfaction). Both of these measurements show similar trends to the error rate (Appendices 2 and 3).

\subsection{Eye-Tracking Metrics}

In what follows, we analyse the time to first glance at the target AOI, the duration of each task, and the number of fixations on target AOIs by the Austrian participants.

For most of the participants, task 1 (navigating to the map; Figure 10) was easy to carry out. The median for the time to first glance was low (3.26s), whereas for the time to complete the tasks the median was $8.92 \mathrm{~s}$. This means that the participants were quick to locate the target AOI and spent $36.5 \%$ of their time searching for it. The rest of their time $(63.5 \%)$ was used for finishing the task. This seems to be unexpectedly high. However, since this was the first interaction with the application, participants had to familiarize themselves with it. This assumption supports the high number of fixations (median = 28.5). Medium-high IT-literate participants were quicker to locate the target AOI and in completing the task compared to low-medium IT-literate participants. This supports the findings of the previous section, since high IT-literate participants finished the tasks with fewer errors. 

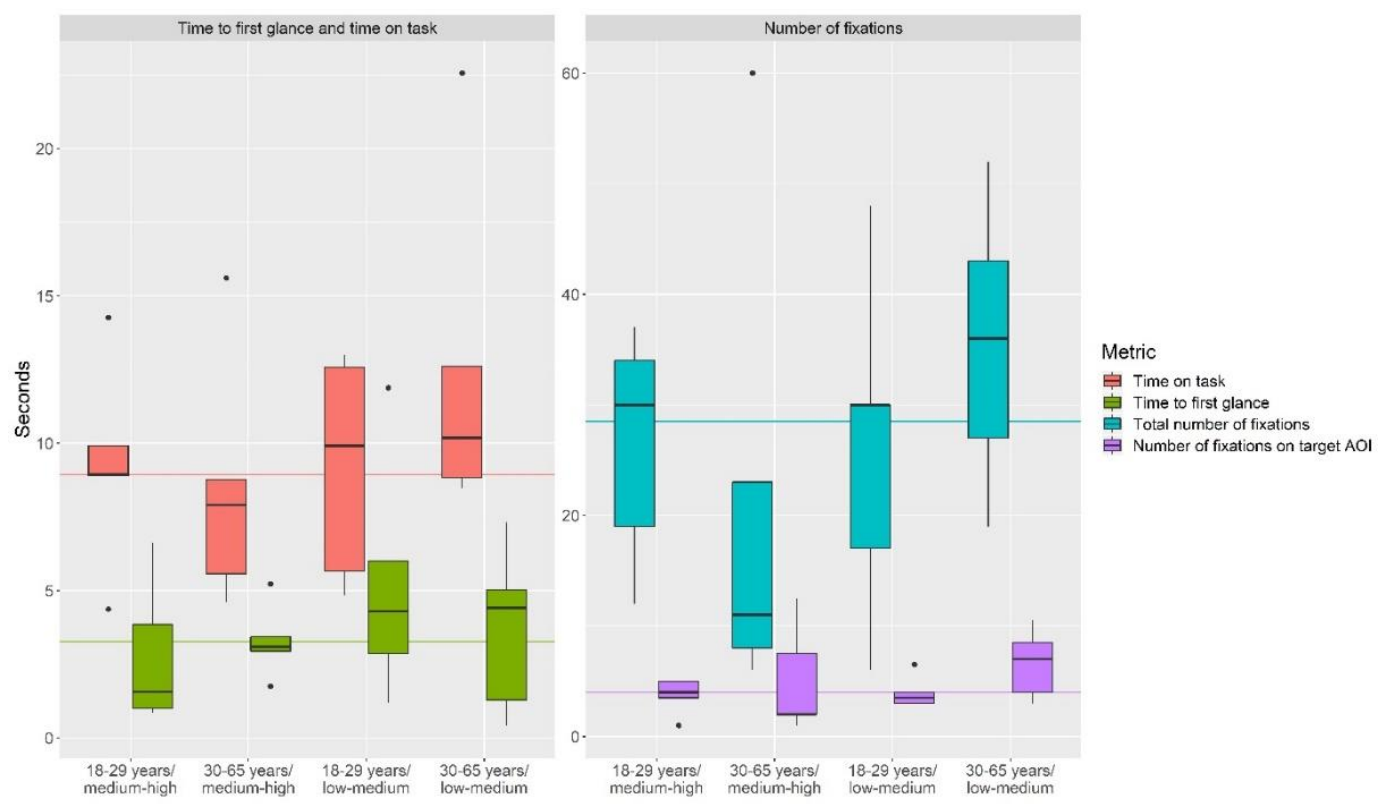

Figure 10: Eye-Tracking Metrics for Task 1 (Navigating to the Map)

For task 2 (changing the basemap; Figure 11), after the first glance at the target AOI, participants spent $81.3 \%$ of the task-completion time on understanding how to carry out the task and making sense of the information. Here, the median time to first glance was $2.02 \mathrm{~s}$, whereas the median task-completion time was $10.83 \mathrm{~s}$. The same trend also supports the number of fixations. The median number of fixations was twice as high (43) as in the previous task, indicating the reduced search-efficiency due to the ambiguity of the button icons. 

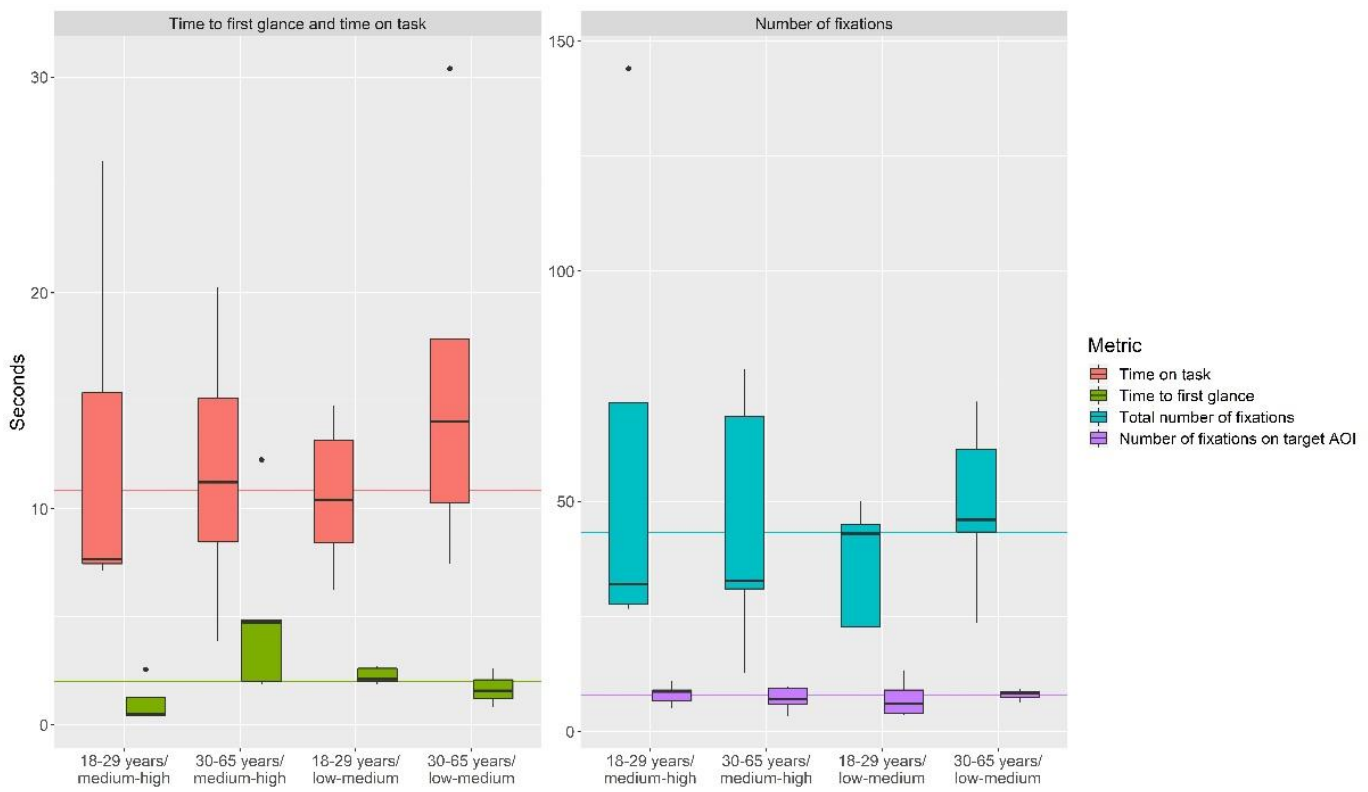

Figure 11: Eye-Tracking Metrics for Task 2 (Changing the Basemap)

Task 3 (to find a POI and review its description; Figure 12) showed the highest medians of all tasks and of all metrics, except for the total number of fixations. The median for the time to first glance was 7.82s, and for the time taken to complete the task it was $34.87 \mathrm{~s}$. Hence, participants spent $22.4 \%$ of their time locating the target AOI and $81.6 \%$ understanding how to carry out the task. The median number of fixations was extremely high (59.5), indicating a reduced search-efficiency. In comparison, the median number of fixations on the target AOIs was low (3.75). The user group of people aged 18-29 with medium-high IT skills had a medium of 0 fixations on the target AOI. As stated in the previous section, participants faced difficulties in locating the search bar, and many participants navigated randomly on the map in order to solve the task. 



Figure 12: Eye-Tracking Metrics for Task 3 (Find a POl and Review its Description)

For task 4 (leaving a comment on an existing POI; Figure 13), we observe a low median for the time taken to complete the task (6.87s). The median for the time to first glance was 3.46s. Participants spent $50.4 \%$ of their time looking for the right AOI. The median number of fixations was 16.33, the lowest for any task. This demonstrates a high search-efficiency. People in the 18-29 group with medium-high IT skills were fast in locating the target AOI and in finishing the task. The older user groups were slower and had a higher numbers of fixations. 


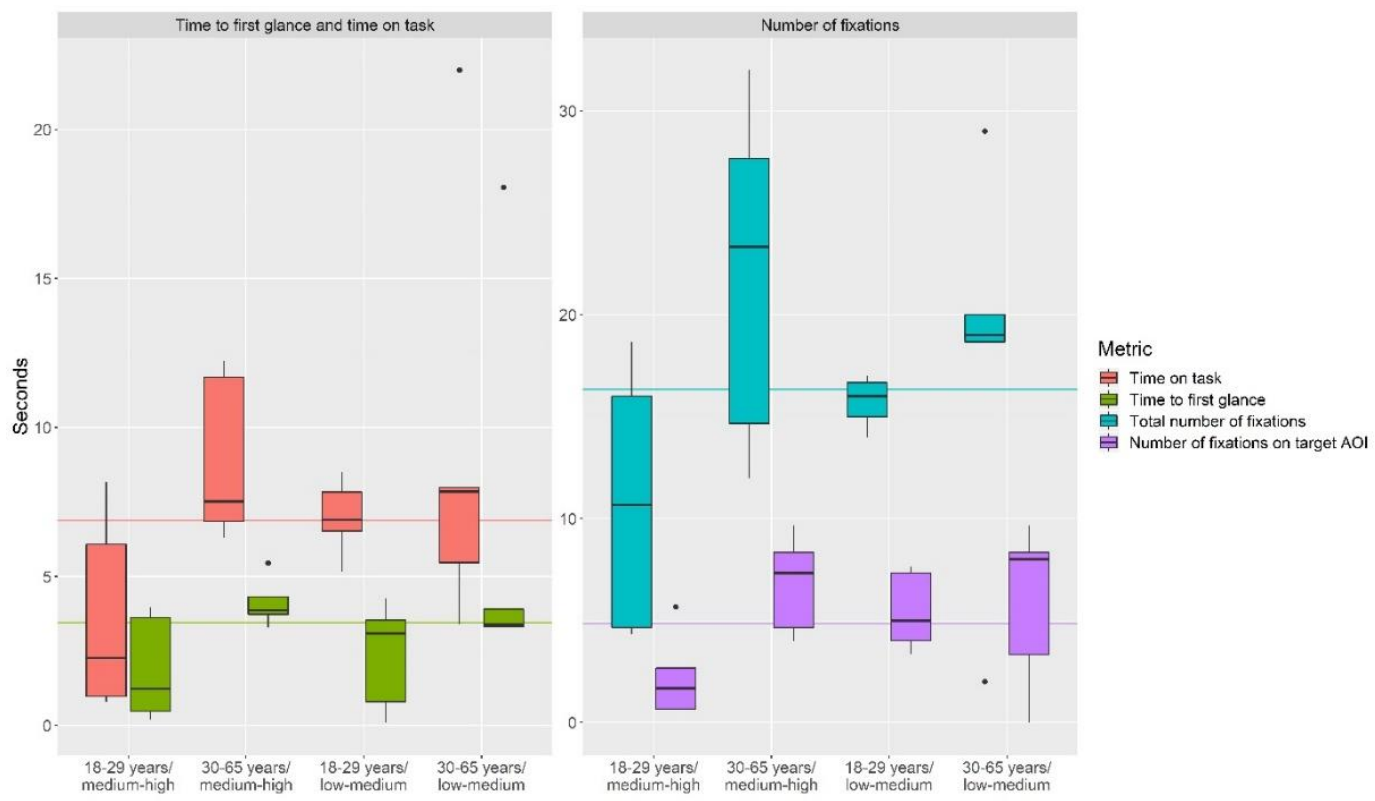

Figure 13: Eye-Tracking Metrics for Task 4 (Leaving a Comment on an Existing POI)

For task 5 (adding a new POI; Figure 14), the median for the time to first glance was 5.04s, and for the time taken to complete the task it was 11.45s. Participants were using $44 \%$ of their time looking for the right AOI and 66\% understanding how to carry out the task. The median number of fixations, 30, was higher than in the previous task, indicating a lower search-efficiency. Low-medium IT-literate participants needed a comparatively long time to finish the task, further indicating a lower efficiency. 

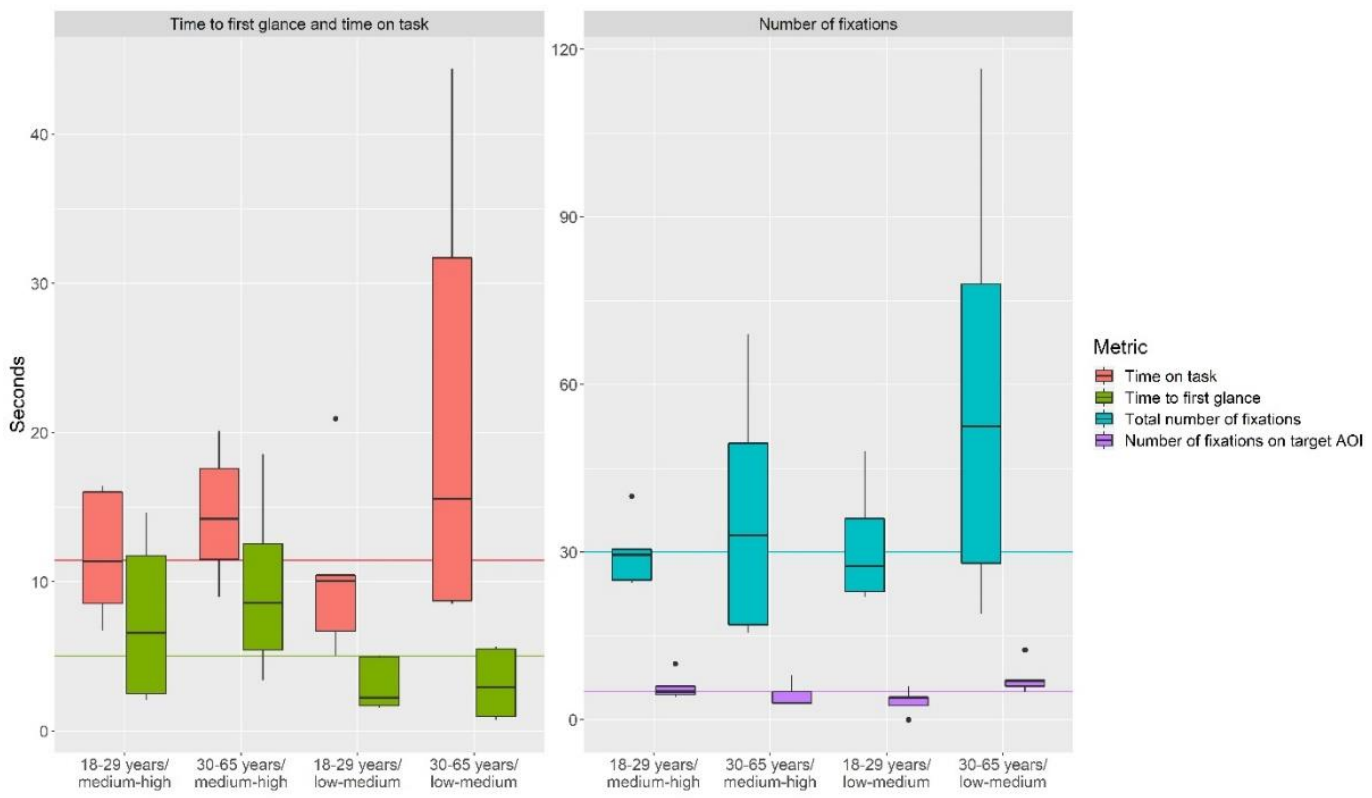

Figure 14: Eye-Tracking Metrics for Task 5 (Adding a new POI)

Since task 6 (locating and changing the profile configuration) was difficult for most of the participants, we excluded it from the eye-tracking data analysis. This was due to difficulties interpreting the data, since participants were searching in different areas of the application to locate the right information.

\section{Discussion and conclusion}

Through the qualitative survey and eye-tracking metrics, we were able to pinpoint individual usability aspects of different functionalities of the PPGIS. We observed that low IT-literate participants showed a higher rate of critical errors in comparison to other user groups. This was due to two factors. First, most of these participants were not accustomed to using an application with functionally complex elements and stated that they typically used their smartphones for social media, messaging applications and phonecalls. Second, most of these participants were not experienced in using map applications. We observed that high ITliterate participants were much more confident in their IT skills and familiar with functionally complex applications; lower IT-literate participants had lower confidence in their skills and were used to more basic applications. For the latter type of participant, we detected a higher level of frustration, with a tendency to give up more easily. This underlines the importance of a positive user-experience for all types of participants.

We also detected differences between the study areas. Most Ugandan participants mentioned not being used to interacting with maps. Low IT-literate participants from Colombia and Austria, by contrast, were more used to interacting with maps, but faced problems with the 
complex functionalities of the application. It is, however, beyond the scope of this article to discuss further differences between the study areas.

Some participants criticized the ambiguity of the button icons, which should be re-designed. Several participants also commented negatively on the non-user-friendly search bar and the zoom and navigation performance of the map. All of this affected the usability of the application, which had an impact on the performance of low IT-literate participants in particular.

We further observed that older user groups and low IT-literate participants were less satisfied than younger and high IT-literate participants. This underlines that younger participants may be more accustomed to using functionally complex applications (they are 'digital natives') in comparison to older participants. Several participants with low-medium IT skills faced problems using the application: they felt uncomfortable using it, due to, for example, the complexity of the information. The majority of the participants in the 30-65 user group with low-medium IT skills indicated the need for more time to familiarize themselves with the application and how to use it. This observation gives important insights into the different requirements of each user group with regard to the content visualization, the complexity of the content, and functionalities.

As a limitation of this study, we faced a challenge with the post-calibration process of the eye-tracking data. Since the eye-tracking device was sensitive to movements, the offset of the eye-tracking data had to be handled. This potentially biased the statistics. Further biasing limitations (among others) were the setting of the study (the 'lab environment'), the chosen number of participants, and the way the user groups were established (e.g. grouping of the participants, and the self-rating of the participants' IT-literacy levels).

To conclude, the purpose of the study was to understand not only the key usability issues of the PPGIS but also the differences in usability for different user groups. We were able to see clear differences between the user characteristics: IT-literacy, age and geographical location. This shows the need to respond to the individual requirements of each user group by distinguishing between them in the application's design. Adaptive interfaces may help provide elements that suit the differing needs of the users (Kiefer, Giannopoulos, Athanasios Anagnostopoulos, Schöning, \& Raubal, 2017). Using adaptive interfaces, allows elements to be simplified, and to reduce both the information load and the number of functionalities, depending on the user. With this study, we want to emphasize the importance of responding to the user's characteristics and individual preferences. We therefore suggest exploring approaches to personalizing map applications (for example context-aware systems, mass customization, recommendation systems, content and interface adaptation etc.). All of this is highly important for advancing the usability of PPGIS applications. 


\section{References}

Atzmanstorfer, K., Eitzinger, A., Marin, B. E., Parra Arteaga, A., Gonzalez Quintero, B., \& Resl, R. (2016). HCI-Evaluation of the GeoCitizen-reporting App for citizen participation in spatial planning and community management among members of marginalized communities in Cali, Colombia. GI_Forum, 1, 117-132. https://doi.org/10.1553/giscience2016_01_s117

Atzmanstorfer, K., Resl, R., Eitzinger, A., \& Izurieta, X. (2014). The GeoCitizen-approach: community-based spatial planning - an Ecuadorian case study. Cartography and Geographic Information Science, 41(3), 248-259. https://doi.org/10.1080/15230406.2014.890546

Brown, M., Sharples, S., Harding, J., Parker, C. J., Bearman, N., Maguire, M., . . Jackson, M. (2013). Usability of Geographic Information: Current challenges and future directions. Applied Ergonomics, 44(6), 855-865. https://doi.org/10.1016/j.apergo.2012.10.013

Butt, M. A., \& Li, S. (2015). Usability evaluation of collaborative PPGIS-GeoCWMI for supporting public participation during municipal planning and management services. Applied Geomatics, 7(3), 139-161. https://doi.org/10.1007/s12518-014-0141-0

Çöltekin, A., Heil, B., Garlandini, S., \& Fabrikant, S. I. (2009). Evaluating the Effectiveness of Interactive Map Interface Designs: A Case Study Integrating Usability Metrics with EyeMovement Analysis. Cartography and Geographic Information Science, 36(1), 5-17. https://doi.org/10.1559/152304009787340197

Corbett, J. M., \& Keller, C. P. (2005). An Analytical Framework to Examine Empowerment Associated with Participatory Geographic Information Systems (PGIS). Cartographica: the International Journal for Geographic Information and Geovisualization, 40(4), 91-102. https://doi.org/10.3138/J590-6354-P38V-4269

Haklay, Mordechai, \& Tobón, C. (2003). Usability evaluation and PPGIS: Towards a user-centred design approach. International Journal of Geographical Information Science, 17(6), 577-592. https://doi.org/10.1080/1365881031000114107

International Organization for Standardization (1992). Ergonomic requirements for office work with visual display terminals (VDTs). (ISO 9241).

Irvin, R. A., \& Stansbury, J. (2004). Citizen Participation in Decision Making: Is It Worth the Effort? Public Administration Review, 64(1), 55-65. https://doi.org/10.1111/j.1540-6210.2004.00346.x

Jordan, P. W. (1998). An introduction to usability. London, Bristol, Pa.: Taylor \& Francis.

Kiefer, P., Giannopoulos, I., Athanasios Anagnostopoulos, V., Schöning, J., \& Raubal, M. (2017). Controllability matters: The user experience of adaptive maps. Geoinformatica : an International Journal on Advances of Computer Science for Geographic Information Systems, 21 (2017), 619-641. Retrieved from http://dx.doi.org/10.1007/s10707-016-0282-x

López-Ornelas, E., Abascal-Mena, R., \& Zepeda-Hernández, J. S. (2013). Geospatial Web Interfaces, Why Are They So "Complicated"? In A. Marcus (Ed.), Design, User Experience, and Usability. Web, Mobile, and Product Design: Second International Conference, DUXU 2013, Held as Part of HCI International 2013, Las Vegas, NV, USA, July 21-26, 2013, Proceedings, Part IV (pp. 231-237). Berlin, Heidelberg: Springer Berlin Heidelberg. https://doi.org/10.1007/978-3-642-39253-5_25

Meng, Y., \& Malczewski, J. (2009). Web-PPGIS Usability and Public Engagement: A Case Study in Canmore, Alberta, Canada. CyberGeo: European Journal of Geography. (Article 483). Retrieved from http:/ / cybergeo.revues.org/22849

Nayebi, F., Desharnais, J.-M., \& Abran, A. (2012). The state of the art of mobile application usability evaluation. In 2012 25th IEEE Canadian Conference on Electrical and Computer Engineering (CCECE) (pp. 1-4). IEEE. https://doi.org/10.1109/CCECE.2012.6334930

Newman, G., Zimmerman, D., Crall, A., Laituri, M., Graham, J., \& Stapel, L. (2010). User-friendly web mapping: Lessons from a citizen science website. International Journal of Geographical Information Science, 24(12), 1851-1869. https://doi.org/10.1080/13658816.2010.490532

Nielsen, J., \& Mack, R. L. (1994). Usability inspection methods. New York: Wiley. 
Poole, A., \& Ball, L. J. (2005). Eye Tracking in Human-Computer Interaction and Usability Research: Current Status and Future. In Prospects", Chapter in C. Ghaoui (Ed.): Encyclopedia of Human-Computer Interaction. Pennsylvania: Idea Group, Inc.

Poplin, A. (2015). How user-friendly are online interactive maps?: Survey based on experiments with heterogeneous users. Cartography and Geographic Information Science, 42(4), 358-376. https://doi.org/10.1080/15230406.2014.991427

Sidlar, C. L., \& Rinner, C. (2007). Analyzing the Usability of an Argumentation Map as a Participatory Spatial Decision Support Tool. URIS A Journal. (19 (1)), 47-55.

Skarlatidou, A., \& Haklay, Muki. (2006). Public web mapping: Preliminary Usability Evaluation. GIS Research UK 2006.

Stivers, C. (2016). The Public Agency as Polis. Administration \& Society, 22(1), 86-105. https://doi.org/10.1177/009539979002200105

Timita, M. (2014). The Usability of a Web Mapping Application with a Million Rows of Data. Retrieved from https://www.gislounge.com/usability-web-mapping-application-million-rowsdata/

Tobón, C., \& Haklay, Mordechai. (2002). Usability Engineering and PPGIS: Towards a Learningimproving Cycle. 


\section{Appendices}

\begin{tabular}{|c|c|c|c|c|}
\hline Metric & Response & $\begin{array}{l}\text { Colombia } \\
(n=30)\end{array}$ & $\begin{array}{l}\text { Uganda } \\
(n=23)\end{array}$ & $\begin{array}{l}\text { Austria } \\
(n=20)\end{array}$ \\
\hline \multirow{2}{*}{ Age } & $18-29$ years & 16 & 10 & 10 \\
\hline & $30-65$ years & 14 & 13 & 10 \\
\hline \multirow{2}{*}{ Gender } & Female & 12 & 9 & 11 \\
\hline & Male & 18 & 14 & 9 \\
\hline \multirow{3}{*}{ Educational level } & Secondary & 17 & 8 & 3 \\
\hline & Undergraduate & 9 & 10 & 7 \\
\hline & Postgraduate & 4 & 5 & 10 \\
\hline \multirow{4}{*}{$\begin{array}{l}\text { Self-rated } \\
\text { skills }\end{array}$} & I don't use the computer & - & 3 & - \\
\hline & Basic & 9 & 9 & 4 \\
\hline & Intermediate & 15 & 11 & 6 \\
\hline & Advanced & 6 & - & 10 \\
\hline \multirow{2}{*}{ Ownership of a smartphone } & Yes & 30 & 22 & 19 \\
\hline & No & - & 1 & 1 \\
\hline \multirow{3}{*}{$\begin{array}{l}\text { Self-rated } \\
\text { skills }\end{array}$} & I need help & 1 & 3 & - \\
\hline & I can carry out basic tasks & 8 & 11 & 3 \\
\hline & $\begin{array}{l}\text { I am sufficiently able to } \\
\text { use it }\end{array}$ & 21 & 9 & 17 \\
\hline \multirow{3}{*}{$\begin{array}{l}\text { Frequency of smartphone } \\
\text { use }\end{array}$} & Sometimes & - & 3 & 1 \\
\hline & Every day & 3 & 1 & - \\
\hline & Several times a day & 27 & 19 & 19 \\
\hline \multirow{3}{*}{$\begin{array}{l}\text { Activities } \\
\text { smartphone }\end{array}$} & Messages and social networks & 30 & 22 & 19 \\
\hline & News & 20 & 13 & 16 \\
\hline & Other applications & 24 & 15 & 19 \\
\hline
\end{tabular}

Appendix 1: User Profile and Number of Participants 
Bartling et al



Appendix 2: Time on Task (Efficiency)

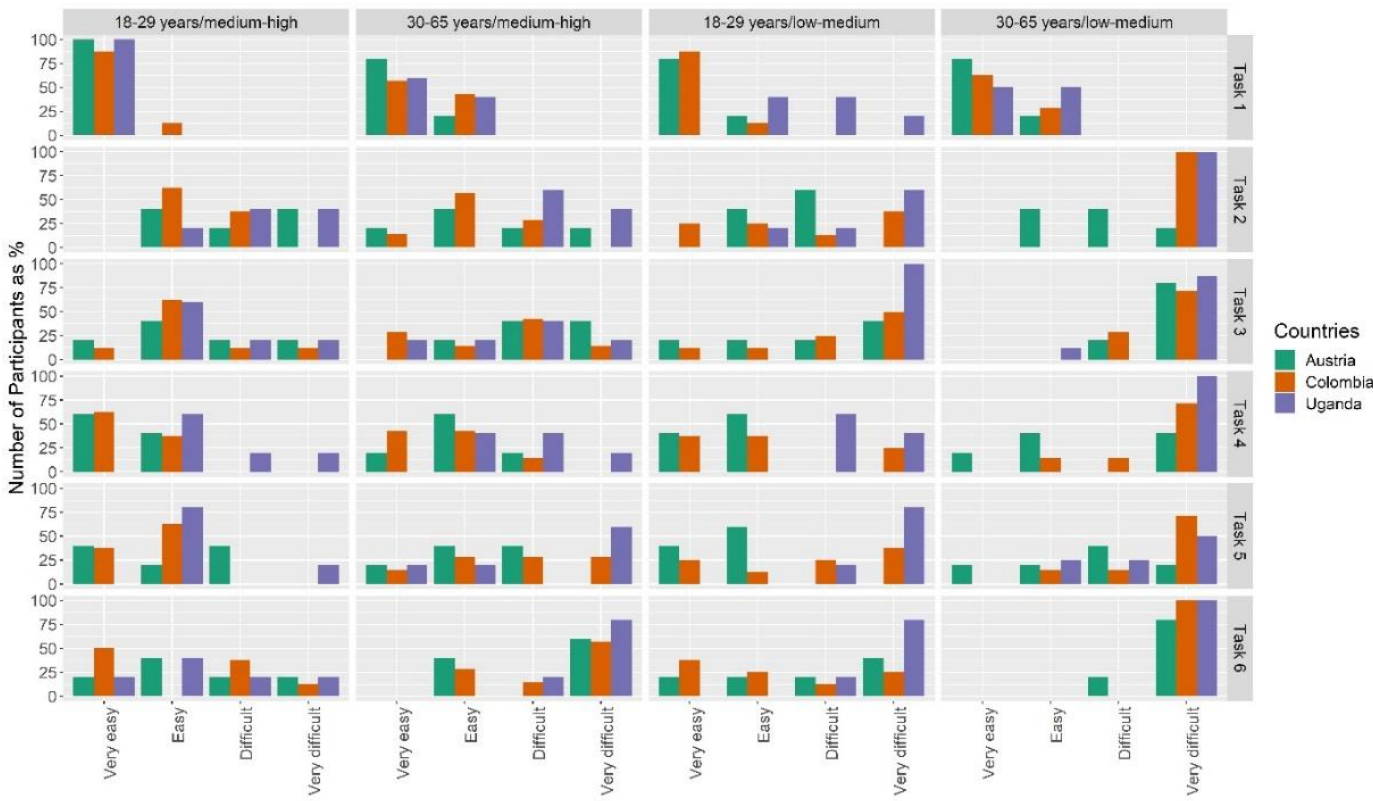

Appendix 3: Task Evaluation by Participants (Satisfaction) 